\title{
VERTICUM-TYPE SYSTEMS APPLIED TO ECOLOGICAL MONITORING
}

\author{
M. Gámez ${ }^{\mathrm{a},}$, I. López ${ }^{\mathrm{a}}$, I. Szabób ${ }^{\mathrm{b}}$ and Z. Varga ${ }^{\mathrm{c}}$ \\ a Departament of Statistics and Applied Mathematics, University of Almería, \\ La Cañada de San Urbano, 04120 Almería, Spain ( mgamez@ual.es, \\ milopez@ual.es) \\ ${ }^{\mathbf{b}}$ Institute of Mechanics and Machinery (Szabo.Istvan@gek.szie.hu) \\ ${ }^{\mathbf{c}}$ Institute of Mathematics and Informatics (Varga.Zoltan@gek.szie.hu) \\ b, c Szent István University, Páter K. u. 1., H-2103 Godollo, Hungary.
}

Abstract. In the paper ecological interaction chains of the type resource - producer primary user - secondary consumer are considered. The dynamic behaviour of these four-level chains is modelled by a system of differential equations, the linearization of which is a verticum-type systems introduced for the study of industrial verticums. Applying the technique of such systems, for the monitoring of the considered ecological system, an observer system is constructed, which makes it possible to recover the whole state process from the partial observation of the ecological interaction chain.

Keywords: ecological chain, stable coexistence, monitoring of an ecosystem, verticumtype system, observer design

\section{Introduction}

Monitoring of ecosystems and management of renewable natural resources are key issues of sustainable human activity. Methodologically both problems can be naturally related to basic concepts of mathematical systems theory such as observability and controllability. The basic theory concerning these concepts was developed in [1] for linear systems (a more recent reference is [2]), however, even the simplest ecosystem models incorporating interacting populations are nonlinear. The corresponding concepts and theorems have been extended with local character to nonlinear systems in [3], but found applications to population systems only recently, see e.g. [4]-[10]. These papers either deal with the theoretical problem of observability or, if also observers are constructed, the results concern only Lotka-Volterra-type population systems.

\footnotetext{
* Corresponding author. Fax: +34-950015167; Phone: +34-950015667

E-mail address: mgamez@ual.es (M. Gámez)
} 
2 In the present paper, observer design for non Lotka-Volterra-type ecosystems will be 3 presented. The ecological model considered in this paper differs from the classical

4 Lotka-Volterra system because of the presence of the dynamics for the resource (or 5 nutrient).

7 Until now in [5], only observability results have been obtained for a trophic chain of resource - producer - primary consumer type. (For a general stability study of multilevel trophic chains see e.g. [11]). In our paper, on the one hand, we extend and modify this model, to deal with a four-level ecological interaction chain of type resource producer - primary user - secondary consumer. Here the term primary user refers to the fact that an animal species is in commensalism with the plant, rather than consuming it. On the other hand, we not only find sufficient conditions for observability, but by the construction of an observer system, we also estimate the state process. From methodological aspect, it will be seen, that the linearization of this four-level ecological interaction chain is a verticum-type system. Systems-theoretical study of such systems was carried out in Molnár [12]-[19], Molnár and Szigeti [20].

The present paper is organized as follows. In Section 2 the dynamic model of the considered four-level ecological chain is set up and its positive equilibrium is calculated. In Section 3 a sufficient condition for the stable coexistence of the ecosystem is proved. Section 4 is devoted to the problem of observability and observer design for the considered ecosystem. The method is also illustrated with a numerical example. In Section 5 a discussion of the results is given. Finally, in the Appendix some basic concepts and results concerning verticum-type systems are shortly recalled.

As a modification of the well-known three-level trophic chain consisting of resource producer - primary consumer considered in [5], we consider the following four-level ecological interaction chain: level 0: a resource; 
1 level 1: the producer is a plant, supposed to die out without the resource, and the positive effect of the latter is proportional to the quantity of the resource present in the system;

level 2: the primary user (instead of consumer), i.e. a commensalist animal, making use of the plant as part of its habitat without harming it (e.g. an insect species hosted by the plant), displaying a logistic dynamics in absence of the plant and the secondary consumer;

level 3: the secondary consumer is a monophagous predator of the primary user (e.g. an insectivorous singing bird species), with intraspecific competition.

(For more details on the role of commensalism in ecological communities, we supposed between the producer and the primary user, see [21]).

For a dynamical model let $x_{0}$ be the time-dependent quantity, with a constant supply $Q$ of the resource present in the system, $x_{1}, x_{2}$ and $x_{3}$ the time-varying population size (biomass or density) of the producer, the primary user and the secondary consumer, respectively. Assume that a unit of biomass of the plant consumes the resource at velocity $\alpha_{0} x_{0}$; however, it increases the biomass of the plant at rate $k_{1}$. The relative rate of increase in biomass of the primary user, due to the presence of the plant is $k_{2} x_{1}$. While the plant population is supposed to die out exponentially in the absence of the resource, with Malthus parameter $m_{1}$, the primary user displays a logistic growth with Malthus parameter $m_{2}$ and is limited by a carrying capacity $\frac{m_{2}}{\eta_{2}}$. Furthermore, the secondary consumer would die out at rate $m_{1}$, without the presence of the primary user, and there is an intraspecific competition among predators with rate $\eta_{3}$. We will consider a partially closed system, where the dead plants may be recycled into nutrient resource with rate $\beta_{1}$. Then with parameters

$$
\left.Q, \alpha_{0}, \alpha_{1}, \alpha_{2}, m_{1}, m_{2}, m_{3}, \eta_{2}, \eta_{3}>0 ; k_{1}, k_{2}, k_{3} \in\right] 0,1\left[; \beta_{1} \in[0,1[,\right.
$$

we have the following dynamic model for the considered interaction chain: 
Verticum-type ecological systems

1

$$
\begin{aligned}
& \dot{x}_{0}=Q-\alpha_{0} x_{0} x_{1}+\beta_{1} m_{1} x_{1} \\
& \dot{x}_{1}=x_{1}\left(-m_{1}+k_{1} \alpha_{0} x_{0}\right) \\
& \dot{x}_{2}=x_{2}\left(m_{2}+k_{2} x_{1}-\eta_{2} x_{2}-\alpha_{2} x_{3}\right) \\
& \dot{x}_{3}=x_{3}\left(-m_{3}+k_{3} \alpha_{2} x_{2}-\eta_{3} x_{3}\right)
\end{aligned}
$$

2 Our purpose now is to find sufficient conditions for the existence of an ecological 3 equilibrium of dynamic system (2.1)-(2.4), where all components are present. The right4 hand side of the system is given by the following function:

5

$$
f: R^{4} \rightarrow R^{4}, f(x)=f\left(x_{0}, x_{1}, x_{2}, x_{3}\right):=\left[\begin{array}{l}
Q-\alpha_{0} x_{0} x_{1}+\beta_{1} m_{1} x_{1} \\
x_{1}\left(-m_{1}+k_{1} \alpha_{0} x_{0}\right) \\
x_{2}\left(m_{2}+k_{2} x_{1}-\eta_{2} x_{2}-\alpha_{2} x_{3}\right) \\
x_{3}\left(-m_{3}+k_{3} \alpha_{2} x_{2}-\eta_{3} x_{3}\right)
\end{array}\right]
$$

6

7 Then a vector $x^{*} \in R^{4}, x^{*}>0$ is an equilibrium for the dynamical system (2.1)-(2.4) if 8 and only if, $f\left(x^{*}\right)=0$. From (2.2) we obviously get

$$
x_{0}^{*}=\frac{m_{1}}{k_{1} \alpha_{0}}>0 .
$$

Since $\left.k_{1} \in\right] 0,1\left[\right.$ and $\beta_{1} \in[0,1[$, from (2.1) we obtain

$$
x_{1}^{*}=\frac{Q}{\alpha_{0} \frac{m_{1}}{k_{1} \alpha_{0}}-\beta_{1} m_{1}}=\frac{Q k_{1}}{m_{1}\left(1-\beta_{1} k_{1}\right)}>0 .
$$

From (2.3) and (2.4), we have

$$
x_{3}^{*}=\frac{k_{3} \alpha_{2}\left(m_{2}+k_{2} \frac{Q k_{1}}{m_{1}\left(1-\beta_{1} k_{1}\right)}\right)-\eta_{2} m_{3}}{k_{3} \alpha_{2}^{2}+\eta_{2} \eta_{3}}>0 \Leftrightarrow k_{3} \alpha_{2}\left(m_{2}+k_{2} \frac{Q k_{1}}{m_{1}\left(1-\beta_{1} k_{1}\right)}\right)-\eta_{2} m_{3}>0 .
$$

It is easy to see that the latter inequality holds, whenever for the model parameters we have

$$
Q>\frac{m_{1} m_{3} \eta_{2}}{k_{1} k_{2} k_{3} \alpha_{2}} .
$$

Indeed, by $\left.k_{1} \in\right] 0,1\left[\right.$ and $\beta_{1} \in[0,1[$, from (2.6) we get 
Verticum-type ecological systems

1

$$
\eta_{2} m_{3}<\frac{k_{1} k_{2} k_{3} \alpha_{2} Q}{m_{1}}<\frac{k_{1} k_{2} k_{3} \alpha_{2} Q}{m_{1}\left(1-\beta_{1} k_{1}\right)}<k_{3} \alpha_{2}\left(m_{2}+k_{2} \frac{Q k_{1}}{m_{1}\left(1-\beta_{1} k_{1}\right)}\right) .
$$

2

3 Finally, under condition (2.6), the positivity of $x_{3}^{*}$ implies

5

6

8

9

10

18

20

$$
\begin{aligned}
& a_{4}=1 \\
& a_{3}=\alpha_{0} x_{1}^{*}+\eta_{2} x_{2}^{*}+\eta_{3} x_{3}^{*} \\
& a_{2}=\left(\eta_{2} \eta_{3}+\alpha_{2}{ }^{2} k_{3}\right) x_{2}^{*} x_{3}^{*}+\alpha_{0} x_{1}^{*}\left(\alpha_{0} k_{1} x_{0}^{*}-\beta_{1} k_{1} m_{1}+\eta_{2} x_{2}^{*}+\eta_{3} x_{3}^{*}\right) \\
& a_{1}=\alpha_{0} x_{1}^{*}\left[\left(\eta_{2} \eta_{3}+\alpha_{2}{ }^{2} k_{3}\right) x_{2}^{*} x_{3}^{*}+k_{1}\left(\eta_{2} x_{2}^{*}+\eta_{3} x_{3}^{*}\right)\left(\alpha_{0} x_{0}^{*}-\beta_{1} m_{1}\right)\right] \\
& a_{0}=\alpha_{0} k_{1}\left(\eta_{2} \eta_{3}+\alpha_{2}{ }^{2} k_{3}\right)\left(\alpha_{0} x_{0}^{*}-\beta_{1} m_{1}\right) x_{1}^{*} x_{2}^{*} x_{3}^{*} .
\end{aligned}
$$

\section{Asymptotic stability of the equilibrium}

For the analysis of stability for the above calculated ecological equilibrium $x^{*}$, let us linearize system (2.1)-(2.4) near $x^{*}$, obtaining

$$
A:=f^{\prime}\left(x^{*}\right)=\left(\begin{array}{cccc}
-\alpha_{0} x_{1}^{*} & -\alpha_{0} x_{0}^{*}+\beta_{1} m_{1} & 0 & 0 \\
k_{1} \alpha_{0} x_{1}^{*} & 0 & 0 & 0 \\
0 & k_{2} x_{2}^{*} & -\eta_{2} x_{2}^{*} & -\alpha_{2} x_{2}^{*} \\
0 & 0 & k_{3} \alpha_{2} x_{3}^{*} & -\eta_{3} x_{3}^{*}
\end{array}\right) .
$$

19

where

$$
\begin{aligned}
& p(\lambda):=\operatorname{det}\left(\begin{array}{cccc}
-\alpha_{0} x_{1}^{*}-\lambda & -\alpha_{0} x_{0}^{*}+\beta_{1} m_{1} & 0 & 0 \\
k_{1} \alpha_{0} x_{1}^{*} & -\lambda & 0 & 0 \\
0 & k_{2} x_{2}^{*} & -\eta_{2} x_{2}^{*}-\lambda & -\alpha_{2} x_{2}^{*} \\
0 & 0 & k_{3} \alpha_{2} x_{3}^{*} & -\eta_{3} x_{3}^{*}-\lambda
\end{array}\right) \\
&=a_{0}+a_{1} \lambda+a_{2} \lambda^{2}+a_{3} \lambda^{3}+a_{4} \lambda^{4},
\end{aligned}
$$


1 Now the well-known Routh-Hurwitz criterion (see e.g. [2]) can be applied: all roots of

2 the polynomial $p(\lambda)=a_{0}+a_{1} \lambda+a_{2} \lambda^{2}+a_{3} \lambda^{3}+a_{4} \lambda^{4}$ have negative real parts if and 3 only if

$$
a_{i}>0 \quad i=0,1, \ldots, 4 \quad ; \quad a_{3} a_{2}>a_{1} a_{4} \text { and } a_{1}\left(a_{3} a_{2}-a_{1} a_{4}\right)>a_{0} a_{3}^{2} \text {. }
$$

For this polynomial we clearly have that $a_{i}>0 \quad i=0,1, \ldots, 4$ since $\left.k_{1} \in\right] 0,1[$ and $\beta_{1} \in\left[0,1\left[\right.\right.$, moreover, $a_{3} a_{2}-a_{1} a_{4}$ can be written as

$$
\begin{aligned}
a_{3} a_{2}-a_{1} a_{4} & =\alpha_{0}{ }^{3} k_{1} x_{0}^{*}\left(x_{1}^{*}\right)^{2}+\left(\eta_{2} \eta_{3}+\alpha_{2}{ }^{2} k_{3}\right) x_{2}^{*} x_{3}^{*}\left(\eta_{2} x_{2}^{*}+\eta_{3} x_{3}^{*}\right)+ \\
& +\alpha_{0} x_{1}^{*}\left(\eta_{2} x_{2}^{*}+\eta_{3} x_{3}^{*}\right)^{2}+\left(\alpha_{0} x_{1}^{*}\right)^{2}\left(-\beta_{1} m_{1} k_{1}+\eta_{2} x_{2}^{*}+\eta_{3} x_{3}^{*}\right)= \\
& =\left(\alpha_{0} x_{1}^{*}\right)^{2}\left(\alpha_{0} k_{1} x_{0}^{*}-\beta_{1} m_{1} k_{1}+\eta_{2} x_{2}^{*}+\eta_{3} x_{3}^{*}\right)+ \\
& +\left(\eta_{2} \eta_{3}+\alpha_{2}{ }^{2} k_{3}\right) x_{2}^{*} x_{3}^{*}\left(\eta_{2} x_{2}^{*}+\eta_{3} x_{3}^{*}\right)+\alpha_{0} x_{1}^{*}\left(\eta_{2} x_{2}^{*}+\eta_{3} x_{3}^{*}\right)^{2},
\end{aligned}
$$

where again by $\beta_{1}, k_{1} \in\left[0,1\left[\right.\right.$ we have that $a_{3} a_{2}-a_{1} a_{4}>0$. Finally, under condition

$Q k_{1} \alpha_{0}>2 m_{1}^{2}$ we have $a_{1}\left(a_{3} a_{2}-a_{1} a_{4}\right)-a_{0} a_{3}^{2}>0$, therefore all inequalities in (3.2) hold.

Now, the above reasoning can be summarized in the following result.

Theorem 3.1. Let us suppose that for given biological parameters, the resource supply is high enough,

$$
Q>\frac{m_{1} m_{3} \eta_{2}}{k_{1} k_{2} k_{3} \alpha_{2}}, \quad Q>\frac{2 m_{1}^{2}}{k_{1} \alpha_{0}} .
$$

Then, both the open $\left(\beta_{1}=0\right)$ and the partially closed $\left(\beta_{1}>0\right)$ ecological chains stably coexist in the sense there exists a positive equilibrium $x^{*}$ of system calculated in Section 2, which is asymptotically stable.

Remark 3.1. The conditions of Theorem 3.1 can also be formulated conversely: Given a resource supply $Q$, biological parameters satisfying conditions (3.3) imply the stable coexistence of the considered ecological chain. 


\section{Observability and observer design}

In this section, in order to address the monitoring problem of the considered ecosystem, first we find sufficient conditions for the observability of the system, reducing the problem, by linearization to the observability of a verticum-type system. Then observer design is used for the asymptotic estimation of the unknown state process, on the basis of a partial observation of the ecosystem.

\subsection{Observability of the ecological chain}

Let us consider now the following two auxiliary 2-dimension systems

$$
\begin{aligned}
& \dot{x}_{0}=Q-\alpha_{0} x_{0} x_{1}+\beta_{1} m_{1} x_{1} \\
& \dot{x}_{1}=x_{1}\left(-m_{1}+k_{1} \alpha_{0} x_{0}\right)
\end{aligned}
$$

and

$$
\begin{aligned}
& \dot{x}_{2}=x_{2}\left(m_{2}+k_{2} x_{1}^{*}-\eta_{2} x_{2}-\alpha_{2} x_{3}\right) \\
& \dot{x}_{3}=x_{3}\left(-m_{3}+k_{3} \alpha_{2} x_{2}-\eta_{3} x_{3}\right)
\end{aligned}
$$

In ecological terms (4.1) is a subsystem of the original chain (2.1)-(2.4), while in (4.2) the positive effect of the plant on the animal species 2 appears with the equilibrium value $x_{1}^{*}$ of the plant. We note that by setting $k_{2}:=0$ (i.e. considering the original system without commensalisms), the original ecological chain is split up into two components without interaction.

Remark 4.1. The biological interpretation of system (4.2) is the following: Suppose that system (2.1)-(2.4) is in equilibrium, and the two animal species, by an external disturbance, deviate from their equilibrium densities. Then the resource-primary consumer subsystem can maintain its equilibrium, and the predator-prey subsystem will be governed by system (4.2).

Continuing the study of systems (4.1) and (4.2), we can easily check that they have respective equilibria $w_{0}^{*}:=\left(x_{0}^{*}, x_{1}^{*}\right)$ and $w_{1}^{*}:=\left(x_{2}^{*}, x_{3}^{*}\right)$. For system (4.1) with notation $w_{0}:=\left(x_{0}, x_{1}\right)$, let us consider observation function

$$
h_{0}\left(w_{0}\right)=h_{0}\left(x_{0}, x_{1}\right):=x_{0}-x_{0}^{*} .
$$


1 This means that the deviation of the resource from its equilibrium value is observed. In

2 order to check local controllability, we calculate the linearization of system (4.1) at 3 equilibrium $w_{0}^{*}$ :

4

$$
A_{00}:=\left(\begin{array}{cc}
-\alpha_{0} x_{1}^{*} & -\alpha_{0} x_{0}^{*}+\beta_{1} m_{1} \\
k_{1} \alpha_{0} x_{1}^{*} & 0
\end{array}\right) \quad ; \quad C_{0}:=h_{0}^{\prime}\left(w_{0}^{*}\right)=\left(\begin{array}{ll}
1 & 0
\end{array}\right)
$$

6

7 Hence we easily calculate

$$
\operatorname{rank}\left(\begin{array}{c}
C_{0} \\
C_{0} A_{00}
\end{array}\right)=2
$$

9 provided $\beta_{1}>0$. From the classical sufficient condition for the local observability of nonlinear systems, [3], we obtain the local observability of system (4.1) near the equilibrium, with observation (4.3).

Similarly, suppose that in system (4.2) the deviation of the density of the prey from its equilibrium value is observed, i.e., with notation $w_{1}:=\left(x_{2}, x_{3}\right)$ we consider the observation function

$$
h_{1}\left(w_{1}\right):=x_{2}-x_{2}^{*} \text {. }
$$

The linearization of system (4.2) at equilibrium $w_{1}^{*}$ is

$$
A_{11}:=\left(\begin{array}{ll}
-\eta_{2} x_{2}{ }^{*} & -\alpha_{2} x_{2}{ }^{*} \\
k_{3} \alpha_{2} x_{3}{ }^{*} & -\eta_{3} x_{3}{ }^{*}
\end{array}\right) \quad ; \quad C_{1}:=h_{1}^{\prime}\left(w_{1}^{*}\right)=\left(\begin{array}{ll}
1 & 0
\end{array}\right) .
$$

Checking again the rank condition, by $\alpha_{2}>0$ we get

$$
\operatorname{rank}\left(\begin{array}{c}
C_{1} \\
C_{1} A_{11}
\end{array}\right)=2
$$

implying local observability of system (4.2), (4.5) near $w_{1}^{*}$.

Now, let us observe that with definition

$$
A_{10}:=\left(\begin{array}{cc}
0 & k_{2} x_{2}^{*} \\
0 & 0
\end{array}\right)
$$

system matrix

$$
A:=\left(\begin{array}{cc}
A_{00} & 0 \\
A_{10} & A_{11}
\end{array}\right)
$$

together with observation matrix 
Verticum-type ecological systems

1

2 define a verticum-type linear observation system in the sense defined in the Appendix.

3

4

5

6

7

8

9

10

Applying Theorem A.1 of the Appendix, we obtain that the linear observation system

$$
\begin{gathered}
\dot{w}=A w \\
y=C w
\end{gathered}
$$

is observable. Since $A$ is just the Jacobian of the righ-hand side of system (2.1)-(2.4) calculated in (3.1), therefore (4.7) is just the linearization system (2.1)-(2.4). Furthermore (4.8) is the linearization of observation function

$$
h(x):=\operatorname{col}\left(x_{0}-x_{0}^{*}, x_{2}-x_{2}^{*}\right)
$$

which can be associated with system (2.1)-(2.4). Finally, applying again the classical rank condition of [3], we can summarize the reasoning of this subsection in the following theorem.

Theorem 4.1. Let us suppose that ecological chain (2.1)-(2.4) is partially closed $\left(\beta_{1}>0\right)$. Then with observation function (4.9), system (2.1)-(2.4) is locally observable near equilibrium $x^{*}$ calculated in Section 2.

\subsection{Construction of an observer system}

Following the procedure of Sundarapandian [22], let us first determine conditions for the construction of observers for systems (4.1) and (4.2), with respective observation functions (4.3) and (4.5).

For matrices $A_{00}$ and $C_{0}$, figuring in (4.4), we have to find a matrix $H_{0}:=\operatorname{col}\left(h_{00}, h_{01}\right)$ such that

$$
A_{00}-H_{0} C_{0}=\left(\begin{array}{cc}
-\alpha_{0} x_{1}^{*}-h_{00} & -\alpha_{0} x_{0}^{*}+\beta_{1} m_{1} \\
k_{1} \alpha_{0} x_{1}^{*}-h_{01} & 0
\end{array}\right)
$$

is a Hurwitz matrix, i.e. all roots of the characteristic polynomial $p_{0}$ of matrix $A_{00}-H_{0} C_{0}$ have real negative parts. It is easy to check that the latter condition is satisfied if and only if the following inequalities hold:

$$
h_{00}>-\alpha_{0} x_{1}^{*}
$$

$$
C:=\left(\begin{array}{cc}
C_{0} & 0 \\
0 & C_{1}
\end{array}\right)
$$


Verticum-type ecological systems

1

2

3

4

5

6

7

9

$$
h_{01}<k_{1} \alpha_{0} x_{1}^{*} .
$$

Simple sufficient conditions for (4.10) and (4.11) are $h_{00}>0$ and $h_{01}<0$, respectively. By the Theorem of Sundarapandian [22], the observer for system (4.1) with observation function (4.3) can be determined.

Similarly, for matrices $A_{11}$ and $C_{1}$, figuring in (4.6), we need to find a matrix $H_{1}:=\operatorname{col}\left(h_{12}, h_{13}\right)$ such that all roots of the characteristic polynomial $p_{1}$ of matrix

$$
A_{11}-H_{1} C_{1}=\left(\begin{array}{ll}
-\eta_{2} x_{2}^{*}-h_{12} & -\alpha_{2} X_{2}^{*} \\
k_{3} \alpha_{2} X_{3}^{*}-h_{13} & -\eta_{3} x_{3}^{*}
\end{array}\right)
$$

have real negative parts.

Now a straightforward checking shows that the latter condition is satisfied if and only if $h_{12}$ and $h_{13}$ satisfy the following inequalities:

$$
\begin{aligned}
& h_{12}>-\eta_{2} x_{2}^{*}-\eta_{3} x_{3}^{*}, \\
& h_{13}<\frac{\left(\eta_{2} x_{2}^{*}+h_{21}\right) \eta_{3} x_{3}^{*}+k_{3} \alpha_{2}{ }^{2} x_{2}^{*} x_{3}^{*}}{\alpha_{2} x_{2}^{*}} .
\end{aligned}
$$

Similarly to the previous case, in order to satisfy conditions (4.12) and (4.13), it is sufficient to set $h_{12}>0$ and $h_{13}<0$, and again by the Theorem of Sundarapandian [22], the observer for system (4.2) with observation function (4.5) can be determined. Finally, based on the above reasoning, it will be easy to prove the following result:

Theorem 4.2. Given

$$
H=\left(\begin{array}{cc}
h_{00} & 0 \\
h_{01} & 0 \\
0 & h_{12} \\
0 & h_{13}
\end{array}\right),
$$

with $h_{00}, h_{12}>0$ and $h_{01}, h_{13}<0$, and function $f$ defined in (2.5) system

$$
\dot{z}=f(z)+H(y-h(z))
$$

is a local exponential observer for system (2.1)-(2.4) with observation equation $y=h(x)$, where $h$ is defined in (4.9).

Proof. Let $p$ be the characteristic polynomial of matrix A-HC with 
Verticum-type ecological systems

1

2

3 Then, it is easy to see that $p=p_{1} \cdot p_{2}$ and therefore, from conditions (4.10)-(4.13) we

4 can conclude that $A-H C$ is a Hurwitz matrix and by the Theorem of Sundarapandian 5 [22] the proof is complete.

6

$7 \quad$ Example 4.1. We consider the following system

$$
\begin{aligned}
& \dot{x}_{0}=2.1-0.2 x_{0} x_{1}+0.2 \cdot 0.4 x_{1} \\
& \dot{x}_{1}=x_{1}\left(-0.4+0.84 \cdot 0.2 x_{0}\right) \\
& \dot{x}_{2}=x_{2}\left(0.25+0.7 x_{1}-0.1 x_{2}-0.4 x_{3}\right) \\
& \dot{x}_{3}=x_{3}\left(-0.1+0.9 \cdot 0.4 x_{2}-0.5 x_{3}\right) .
\end{aligned}
$$

10

System (4.14) has a positive equilibrium $x^{*}=(2.38,5.3,10.41,7.3)$, which is asymptotically stable, because conditions of Theorem 3.1 are satisfied. In Fig. 1 it can be seen how, e.g. from initial condition $x(0):=(1,6,9,5)$ near the equilibrium, the solution $x$ of system (4.14) tends to this positive equilibrium, see Fig. 1.

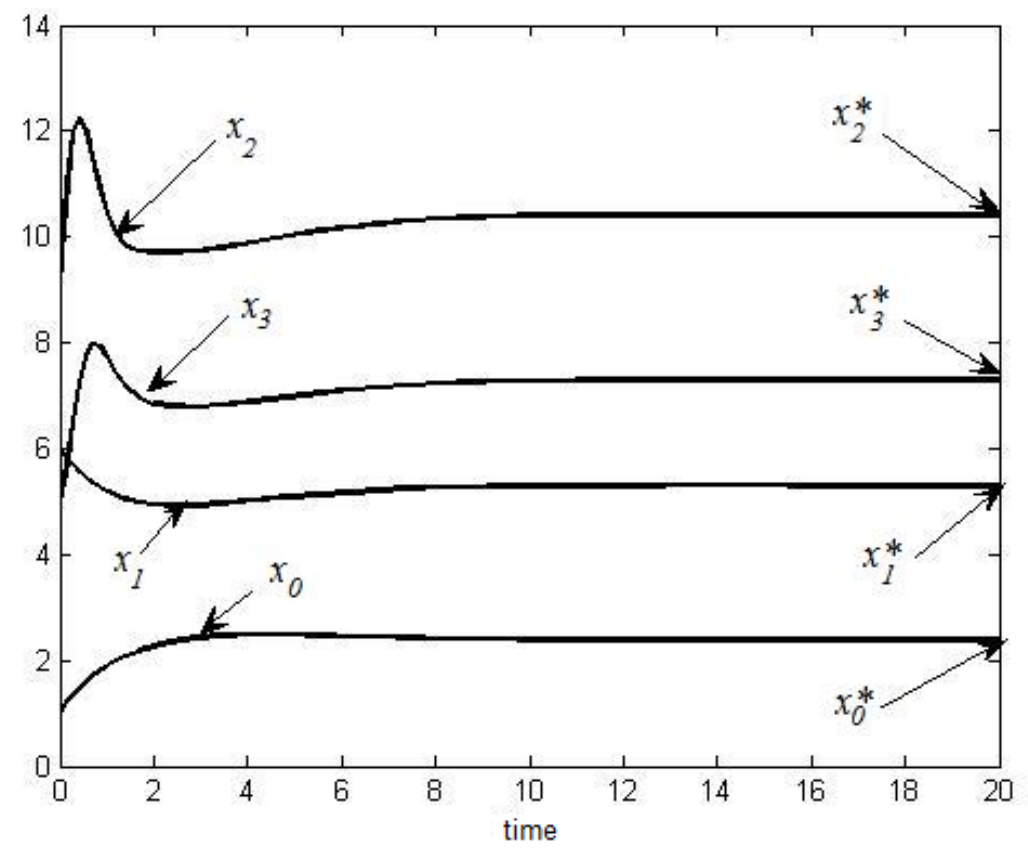

Fig. 1. Solution of system (4.14) with initial condition $x(0)=(1,6,9,5)$

Consider now system (4.14) with observation 
Verticum-type ecological systems

1

2 Since matrix

3

4

8

6 7

8

$$
\begin{aligned}
& \dot{z}_{0}=2.1-0.2 z_{0} z_{1}+0.2 \cdot 0.4 z_{1} \\
& \dot{z}_{1}=z_{1}\left(-0.4+0.84 \cdot 0.2 z_{0}\right) \\
& \dot{z}_{2}=z_{2}\left(0.25+0.7 z_{1}-0.1 z_{2}-0.4 z_{3}\right) \\
& \dot{z}_{3}=z_{3}\left(-0.1+0.9 \cdot 0.4 z_{2}-0.5 z_{3}\right)
\end{aligned}+\left(\begin{array}{cc}
10 & 0 \\
-20 & 0 \\
0 & 10 \\
0 & -0.01
\end{array}\right) \cdot(y-h(z))
$$

9

$$
y=h(x)=\left(x_{0}-x_{0}^{*}, x_{2}-x_{2}^{*}\right)
$$

$$
H=\left(\begin{array}{cc}
10 & 0 \\
-20 & 0 \\
0 & 10 \\
0 & -0.01
\end{array}\right)
$$

satisfies the conditions of Theorem 4.2, we can construct the following observer

(1)

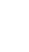

Solving (4.15) with initial condition $z(0)=(2,4,7,7)$ near to the equilibrium, we can check how this solution tends to recover the corresponding solution of system (4.14), see Fig. 2.

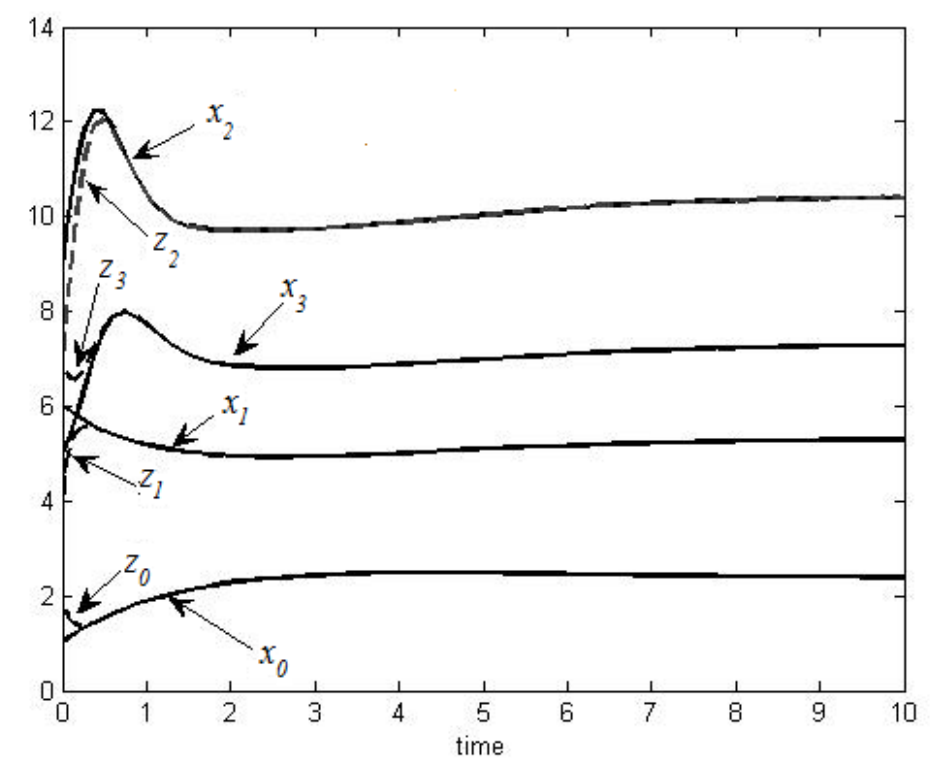

Fig. 2. Solutions of system (4.14) and (4.15) with the respective initial conditions $x(0)=(1,6,9,5)$ and $z(0)=(2,4,7,7)$ 
Verticum-type ecological systems

\section{Discussion}

From the ecological point of view, in comparison to the existing results, the novelty of the paper consists in extending the state estimation (monitoring) from three-level trophic chains to four-level ecological chains, where, a non-trophic interaction (namely commensalism) also takes place. From the methodological aspect, this the first time that by the linearization of the underlying nonlinear dynamic ecosystem model, the technique of verticum-type linear systems (developed for the investigation of industrial systems) is applied to the monitoring of specially structured (chain-type) ecosystems. Finally, we note that the proposed method can be extended to a longer, five-level ecological chain by adding a tertiary consumer (a top predator, e.g. a predator bird consuming the singing bird) to the model.

The considered model can be extended by adding a tertiary consumer (a top predator, e.g. a predator bird consuming the singing bird).

Acknowledgement The research was also supported by the Hungarian National Scientific Research Fund (OTKA 62000 and 68187), the Ministry of Education and Science of Spain, project No. TIN2007-67418-C03-02, and a bilateral project funded by the Scientific and Technological Innovation Fund (of Hungary) and the Ministry of Education and Sciences (of Spain), grant No. HH2008-0023.

\section{References}

[1] R.E. Kalman, P.L. Falb, M. Arbib, Topics in Mathematical System Theory. McGraw-Hill, New York, 1969.

[2] B.M. Chen, Z. Lin, Y. Shamesh, Linear Systems Theory. A Structural Decomposition Approach. Birkhauser, Boston, 2004.

[3] E.B. Lee, L. Markus, Foundations of Optimal Control Theory. Wiley, New YorkLondon-Sydney, 1971. 
1 [4] Z. Varga, A. Scarelli, A. Shamandy, State monitoring of a population system in changing environment. Community Ecology 4 (1): 73-78 (2003).

[5] A. Shamandy, Monitoring of trophic chains. Biosystems 81 (1): $43-48$ (2005).

[6] I. López, M. Gámez, S. Molnár, Observability and observers in a food web. Applied Mathematics Letters 20 (8): 951-957 (2007).

[7] I. López, M. Gámez, J. Garay, Z. Varga, Monitoring in a Lotka-Volterra model. Biosystems 83: 68-74 (2007).

[8] M. Gámez, I. López, Z. Varga, Iterative scheme for the observation of a competitive Lotka-Volterra system. Applied Mathematics and Computation 201: 811-818 (2008).

[9] M. Gámez, I. López, S. Molnár, Monitoring environmental change in an ecosystem. Biosystems 93: 211-217 (2008).

[10] Z. Varga, Applications of mathematical systems theory in population biology. Periodica Mathematica Hungarica 51 (1): 157-168 (2008).

[11] Y.M. Svirezhev, D.O. Logofet, Stability of biological communities. Mir Publishers, Moscow, 1983.

[12] Molnár, S., Model runs for the definition of the most advantageous integrated energetical verticum in the national economy. Publications of Central Mining Development Institute, 30: 121-127 (1987).

[13] S. Molnár, Realization of Verticum-Type Systems. Math. Anal. and System Theory, 5: 11-30, (Karl Marx University of Economics, Budapest) (1988).

[14] S. Molnár, Optimization of Realization-Independent Cost Functions. Math. Anal. and System Theory, 5: 1-10, (Department of Mathematics, Marx Károly University of Economics, Budapest) (1988).

[15] S. Molnár, Observability and Controllability of Decomposed Systems I. Math. Anal. and System Theory, 5: 57-66, (Department of Mathematics, Karl Marx University of Economics, Budapest) (1988).

[16] S. Molnár, Observability and Controllability of Decomposed Systems II. Math. Anal. and System Theory, 5: 67-72, (Department of Mathematics, Karl Marx University of Economics, Budapest) (1988). 
1 [17] S. Molnár, Observability and Controllability of Decomposed Systems III. Math. Anal. and System Theory, 5: 73-80, (Department of Mathematics, Karl Marx University of Economics, Budapest) (1988).

[18] S. Molnár, A special decomposition of linear systems. Belgian Journal of Operations Research, Statistics and Computer Science, 29 (4): 1-19 (1989).

6 [19] S. Molnár, Stabilization of verticum-type systems, Pure Mathematics and Applications, 4 (4): 493-499 (1993).

[21] P.J. Morin, P. Morin, Community Ecology. Wiley-Blackwell, 1991.

[22] V. Sundarapandian, Local observer design for nonlinear systems. Mathematical and computer modelling 35: 25-36 (2002).

In this section, based on [18], we summarize some concepts, notation and a basic sufficient condition for observability of verticum-type systems, in a simplified form used in the present paper. Let $k, n_{i}, r_{i} \in \mathbf{N}(i \in \overline{0, k})$ and assume that

$$
A_{00} \in R^{n_{0} \times n_{0}}, C_{0} \in R^{r_{0} \times n_{0}}
$$

and for all $i \in \overline{0, k}$

$$
A_{i, i-1} \in R^{n_{i} \times n_{i-1}}, A_{i i} \in R^{n_{i} \times n_{i}}, C_{i} \in R^{r_{i} \times n_{i}} .
$$

Consider systems

$$
\begin{aligned}
& \dot{x}_{0}=A_{00} x_{0} \\
& y_{0}=C_{0} x_{0},
\end{aligned}
$$

and for all $i \in \overline{1, k}$

$$
\begin{aligned}
& \dot{x}_{i}=A_{i i} x_{i}+A_{i i-1} x_{i-1} . \\
& y=C_{i} x_{i}
\end{aligned}
$$


1 Denoting $n:=\sum_{i=0}^{k} n_{i}, r:=\sum_{i=0}^{k} r_{i}$, define the matrices $A \in R^{n \times n}, C \in R^{r \times n}$ as follows:

$$
A=\left(\begin{array}{ccccccc}
A_{00} & 0 & 0 & \ldots & 0 & \ldots & 0 \\
A_{10} & A_{11} & 0 & \ldots & 0 & \ldots & 0 \\
0 & A_{21} & A_{22} & \ldots & 0 & \ldots & 0 \\
\cdot & . & . & \ldots & . & \ldots & . \\
. & . & . & \ldots & . & \ldots & . \\
. & . & . & \ldots & . & \ldots & . \\
0 & 0 & 0 & \ldots & . & A_{k-1, k-1} & 0 \\
0 & 0 & 0 & \ldots & . & A_{k, k-1} & A_{k, k}
\end{array}\right)
$$

$$
C=\left(\begin{array}{lllll}
C_{1} & & & & 0 \\
& C_{2} & & & \\
& & \cdot & & \\
& & & \cdot & \\
0 & & & & C_{k}
\end{array}\right) .
$$

\section{Definition A.1. System}

5

$$
\begin{aligned}
& \dot{x}=A x \\
& y=C x
\end{aligned}
$$

6 is said to be of verticum type.

7 Given the verticum-type system (V), using the above notation, let $i, j \in \overline{0, k}$ with $8 \quad j \leq j$, and define system

9

$\left(\mathrm{V}_{\mathrm{ij}}\right)$

$$
\begin{aligned}
& \dot{x}_{i}=A_{i i} x_{i} \\
& \dot{x}_{l}=A_{l l-1} x_{i-1}+A_{l l} x_{l} \quad(l \in i+1, j, \text { if } i<j),
\end{aligned}
$$

10 with observation matrix

$$
C_{i j}:=\left(\begin{array}{ccccc}
C_{i} & & & & 0 \\
& C_{i+1} & & & \\
& & \cdot & & \\
& & & \cdot & \\
0 & & & & C_{j}
\end{array}\right) .
$$

12 Remark A.1. Intuitively, a verticum-type system consists of a finite series of 13 "subsystems" where each "subsystem" is connected only with the previous one. 
Verticum-type ecological systems

1 Theorem A.1. For given $s \in \overline{1, k}$, let $i_{p}, j_{p} \in \overline{0, k}$ with $i_{p} \leq j_{p} \quad(p \in 1, s)$, and suppose

2 that all systems $\mathrm{V}_{\mathrm{i}_{\mathrm{p}} \mathrm{j}_{\mathrm{p}}}$ with observation matrix $C_{i_{p} j_{p}}$ are observable. Then the verticum-

3 type system (V) is also observable. 